\section{Selective suppression of two-photon fluorescence in laser scanning microscopy by ultrafast pulse-train excitation}

\author{
Arijit Kumar De*, Debjit Roy, and Debabrata Goswami \\ Indian Institute of Technology Kanpur, Department of Chemistry, \\ UP-208016, India
}

\begin{abstract}
Selective excitation of a particular fluorophore in the presence of others demands clever design of the optical field interacting with the molecules. We describe the use of 20- to $50-\mathrm{GHz}$ pulse-train excitation leading to two-photon absorption, followed by successive one-photon stimulated emission as a potential technique in the context of controlling two-photon molecular fluorescence, with applications in microscopy. @ 2010 Society of Photo-Optical Instrumentation Engineers. [DOI: 10.1117/1.3509383]
\end{abstract}

Keywords: two-photon absorption; stimulated emission; pulse-pair excitation; pulse-train excitation.

Paper 10391LR received Jul. 10, 2010; revised manuscript received Oct. 1, 2010; accepted for publication Oct. 8, 2010; published online Dec. 23, 2010.

\section{Introduction}

One of the present day challenges in fluorescence microscopy lies in having better depth resolution as embodied by the implementation of multiphoton excitation in laser scanning microscopy. ${ }^{1}$ However, this elegant technique bears its own limitations, having a low nonlinear photon absorption cross section as well as simultaneous excitation of many fluorophores [due to the broad overlapping two-photon absorption (TPA) spectra of fluorophores and the large spectral bandwidth of a short pulse with high instantaneous peak power needed to circumvent the low cross section]. The first issue has been investigated by various groups, including ours, with the design of novel chromophores with large nonlinear absorption. ${ }^{2,3}$ The second issue (i.e., selective enhancement/suppression of fluorescence from individual fluorophores) has been addressed by several groups from the perspective of coherent quantum control $^{4}$ through laser pulse shaping ${ }^{5}$ with applications in fluorescence microscopy. ${ }^{6-8}$ An alternative scheme of controlling spontaneous fluorescence is achieved through coherent wavepacket interference using pulse-pair ${ }^{9}$ and pulse-train ${ }^{10}$ excitation schemes. However, control can also be achieved beyond coherence time scales using stimulated emission, as recently shown

*Currently at: Lawrence Berkeley National Laboratory, Physical Biosciences Division, 1 Cyclotron Road, Berkeley, California 94720.

Address all correspondence to: Debabrata Goswami, Department of Chemistry, Indian Institute of Technology Kanpur, Kanpur, Uttar Pradesh 208016, India. Tel: +91-512-2597187; Fax: +91-512-2597554; E-mail: dgoswami@iitk.ac.in by our group. ${ }^{11,12}$ Extending the previous work using pulse-pair excitation, here we demonstrate how 20- to 50-GHz pulse-train excitation can selectively suppress fluorescence from one particular fluorophore by stimulated emission, which is otherwise not possible using a pulse-pair scheme.

\section{Methodologies}

We used $\sim 160$-fs pulsed excitation, centered on $750 \mathrm{~nm}$, at $76-\mathrm{MHz}$ repetition rate from a mode-locked Ti:sapphire laser (Mira900-F pumped by Verdi5, Coherent, Santa Clara, California). For pulse-pair excitation, we used a collinear MachZehnder-type interferometer described elsewhere and repeated our earlier experiment. ${ }^{11}$ We generated the pulse train with a Fabry-Perot etalon made of a pair of 50/50 beamsplitters $3 \mathrm{~mm}$ thick [Castech (Hamilton, New Zealand) BSP-254-030-780]: Due to multiple reflections between the coated surfaces, each pulse generates a pulse train as shown in Fig. 1(a); the intensity of the successive pulses decreases in geometric progression. The resultant pulse train was correlated with a reference pulse in a collinear interferometer setup to get the field autocorrelation traces. As shown in Fig. 1(b), when the distance between the two coated surfaces was increased by a factor of two, the time delay between two consecutive pulses is also doubled. We mounted one of the beamsplitters on a mechanical stage (UE1724SR driven by ESP300, Newport, Irvine, California) interfaced with a personal computer through a GPIB card (National Instruments, Austin, Texas). The collimated laser beam was sent to a multiphoton-ready confocal microscope system (FV300 scanhead coupled with IX71 inverted microscope, Olympus). The delay between the successive pulses in a pulse train was varied from 20 ps (corresponding to the minimum possible delay constrained by the physical separation between two mounts holding the beamsplitters) up to $50 \mathrm{ps}$ in 1-ps step size, and we collected an image at every step. For imaging purposes, slides of bovine pulmonary artery endothelial (BPAE) cells having nuclei stained with DAPI, and mitochondria stained with Mito Tracker Red CMX Ros (F36924, Molecular Probes, Invitrogen, Carlsbad, California) were used and all images were taken using an oil-immersion objective (UPlanApoN 40×1.4 NA, Olympus). The image acquisition and intensity counts were performed using Fluoview software.

\section{Results and Discussion}

As shown in Fig. 2, the fluorescence from DAPI and Mito Tracker Red decreases to different extents as a function of the interpulse delay, depending on whether a pulse-pair or pulse-train excitation scheme is implemented. Under pulse-train excitation, at 50-ps interpulse separation, the Mito Tracker fluorescence drops to $90 \%$ of that at $20 \mathrm{ps}$, while the DAPI fluorescence is hardly suppressed. This suppression is not noticed under pulse-pair excitation. The corresponding images, shown in Figs. 3(a) and 3(b), reveal this selective fluorescence suppression. However, the effect is not visually very prominent, as the $\sim 10 \%$ fluorescence drop is hardly noticeable.

1083-3668/2010/15(6)/060502/3/\$25.00 @ 2010 SPIE 


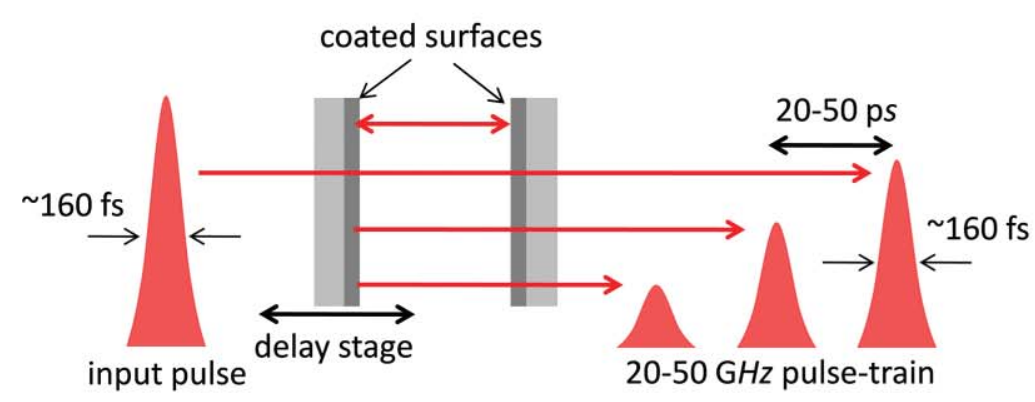

(a)

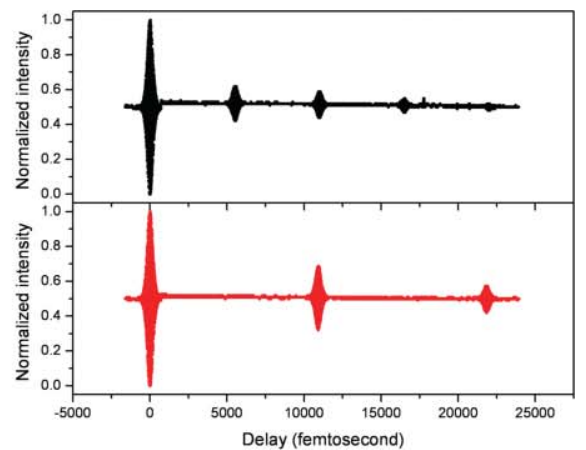

(b)

Fig. 1 (a) Generation of 20- to 50-GHz pulse train used in the experiment, and (b) field autocorrelation traces of a pulse train correlated with a reference pulse. The zero delay refers to arrival of the first pulse in the pulse train. The distance between the successive pulses of the pulse train shown in the lower panel is twice that of the upper panel.

Nevertheless, the present work shows that the pulse-train excitation scheme is a better choice over the pulse-pair one for TPA followed by one-photon selective fluorescence suppression.

Stimulated emission depletion (STED) has been used for imaging beyond the fundamental diffraction limit. ${ }^{13,14}$ Pulsepair excitation leading to stimulated emission has been used to study ultrafast dynamics in microscopy. ${ }^{15}$ In stimulated emission, the first pulse, wavelength-tuned to the one-photon absorption maximum, excites the ground state population to higher vibrational manifolds of the excited electronic state, which is followed by a rapid relaxation to the ground vibrational state of the same electronic state. A time-delayed second pulse, wavelength-tuned to the red-edge fluorescence, dumps the population from this state to the higher vibrational manifolds of the ground electronic state. In contrast, we used pulses centered on one particular wavelength.

For Mito Tracker Red (one-photon absorption maximum $\sim 550 \mathrm{~nm}$ ), the first pulse in a pulse train induces TPA (at $\sim 750 \mathrm{~nm}$ ) from the ground electronic state to a higher excited electronic state (which relaxes to a lower excited elec- tronic state from which fluorescence occurs). The time-delayed successive pulses can either cause TPA or one-photon stimulated emission (from the lower excited electronic state to the ground electronic state). Now, as the energy of the successive pulses decreases in a geometric progression, both the probability of TPA as well as stimulated emission are decreased. However, the decrease of TPA is more, since it depends on the square of the intensity of the laser pulses, while stimulated emission, being a one-photon process, depends linearly on the laser pulse intensity. This is schematically shown in Fig. 3(c). The greater the number of successive pulses, the more pronounced the stimulated emission; thus, pulse-train excitation renders better fluorescence suppression than pulse-pair excitation. An analogy may be drawn with "batch extraction" where a solute is partitioned in two different solvents; the solute is extracted more efficiently when the extracting solvent is used in multiple volumes (i.e., in small amounts every time) than using the entire volume at a single time.

The work presented here describes a better depletion effect of pulse-train excitation over pulse-pair excitation,

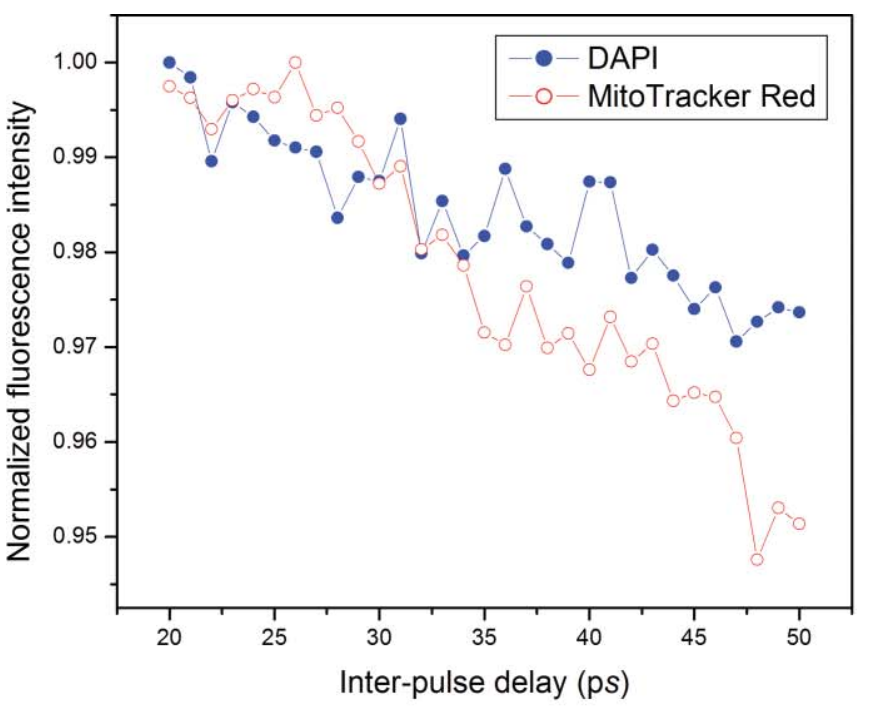

(a)

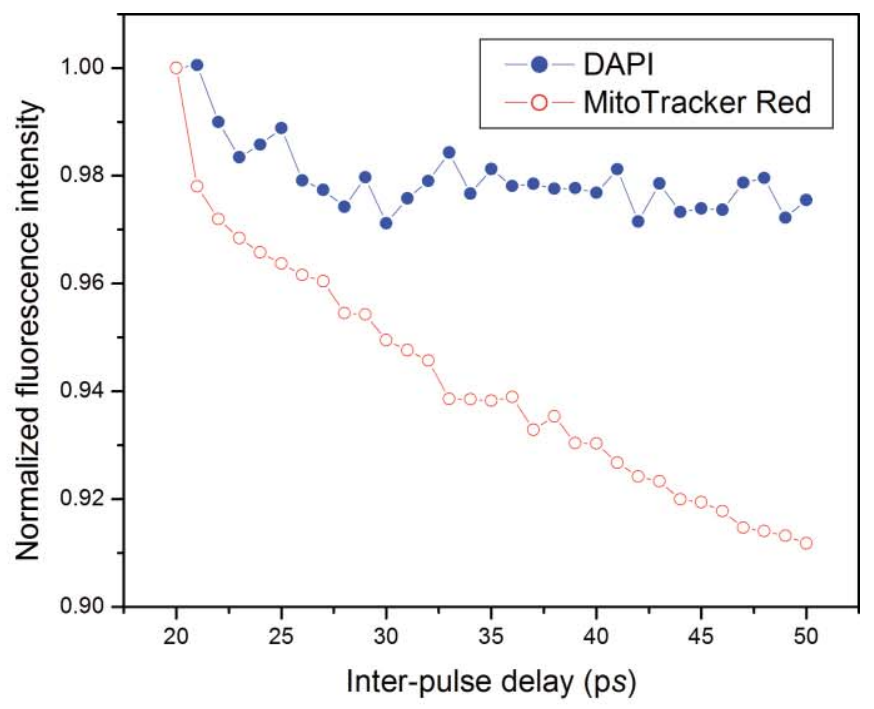

(b)

Fig. 2 Normalized fluorescence intensity of DAPI (blue solid circles) and Mito Tracker Red (red empty circles) as a function of (a) interpulse delay under pulse-pair and (b) pulse-train excitation. (Color online only.) 


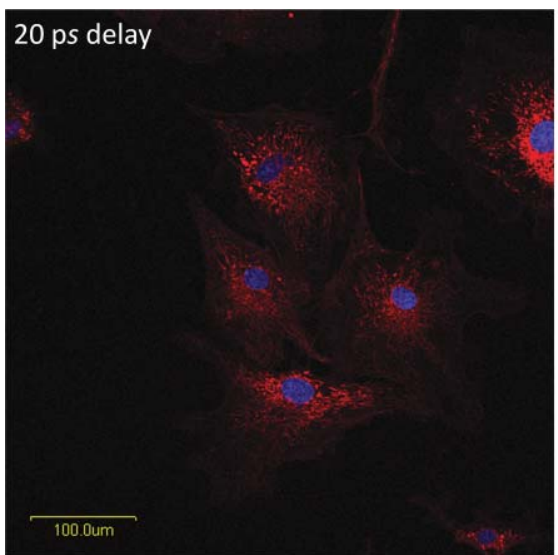

(a)

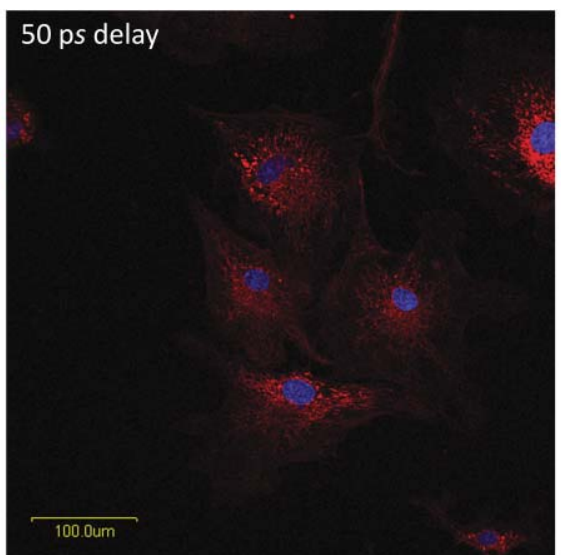

(b)

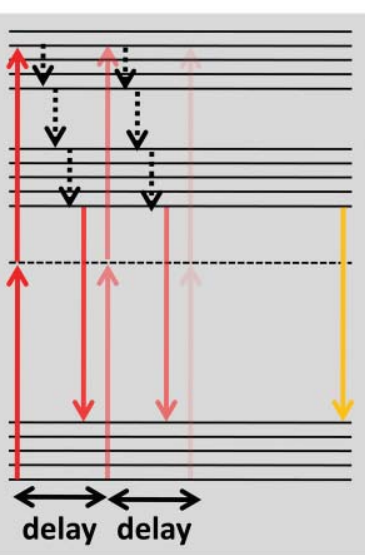

(c)

Fig. 3 Images of BAPE cells showing nuclei (blue) and mitochondria (red) at (a) 20-ps and (b) 50-ps interpulse delay under pulse-train excitation. (c) Schematic of fluorescence (orange arrow) suppression of Mito Tracker Red by stimulated emission (downward red arrows) under pulse-train excitation (upward red arrows); non-radiative relaxation (vibrational relaxation and internal conversion) is shown by dashed black arrows. (Color online only.)

which leads to selective fluorescence suppression. Thus, while pulse-pair excitation renders no suppression of Mito Tracker Red fluorescence, the pulse-train excitation does suppress, albeit with little $(\sim 10 \%)$ contrast enhancement. An analysis after removal of background, arising due to the everpresent backscattered light as well as detector dark noise, shows increased suppression. The importance of the present work lies in the experimental demonstration of the fact that, as in coherent control approaches, ${ }^{4,6-8}$ selective fluorescence suppression can also be achieved by exploiting incoherent dynamics using a much simpler one-color experimental scheme. Note that since the optimally shaped pulse aimed at selective two-photon fluorescence in quantum control schemes always has larger time width compared to the transform-limited pulse, the suppression of fluorescence from both the fluorophores results only in different extents. However, in the present case, due to the use of transform-limited pulses only, fluorescence from only one of the fluorophores is suppressed, keeping the fluorescence from the other fluorophores unaffected.

\section{Conclusions}

In summary, we show how ultrafast laser pulse-train excitation at a high repetition rate can lead to control fluorescence dynamics in microscopy. We demonstrate that pulse-train excitation can be a better choice over pulse-pair excitation when considering a two-photon pump followed by one-photon stimulated emission type control experiments using a single laser source.

\section{Acknowledgments}

We thank DST, India, and Wellcome Trust Foundation, United Kingdom, for funding. De and Roy thank CSIR, India, for graduate fellowships.

\section{References}

1. W. Denk, J. H. Strickler, and W. W. Webb, "Two-photon laser scanning fluorescence microscopy," Science 248, 73-76 (1990).
2. G. S. He, L S. Tan, Q. Zheng, and P. N. Prasad, "Multiphoton absorbing materials: molecular designs, characterizations, and applications," Chem. Rev. 108, 1245-1330 (2008).

3. A. Jana, A. K. De, A. Nag, D. Goswami, and P. K. Bharadwaj, "Diaza-18-crown-6 based chromophores for modulation of two-photon absorption cross-section by metal ions," J. Organometall. Chem. 693, 1186-1194 (2008).

4. T. Brixner, N. H. Damrauer, P. Niklaus, and G. Gerber, "Photoselective adaptive femtosecond quantum control in the liquid phase," Nature 414, 57-60 (2001).

5. D. Goswami, "Optical pulse shaping approaches to coherent control," Phys. Rep. 374, 385-481 (2003).

6. I. Pastirk, J. M. Dela Cruz, K. A. Walowicz, V. V. Lozovoy, and M. Dantus, "Selective two-photon microscopy with shaped femtosecond pulses," Opt. Express 11, 1695-1701 (2003).

7. J. M. Dela Cruz, I. Pastirk, M. Comstock, V. V. Lozovoy, and M. Dantus, "Use of coherent control methods through scattering biological tissue to achieve functional imaging," Proc. Natl. Acad. Sci. USA 101, 16996-17001 (2004).

8. J. Ogilvie, D. Debarre, X. Solinas, J. L. Martin, E. Beaurepaire, and M. Joffre, "Use of coherent control for selective two-photon fluorescence microscopy in live organisms," Opt. Express 14, 759-766 (2006).

9. N. F. Scherer, R. J. Carlson, A. Matro, M. Du, A. J. Ruggiero, V. Romero-Rochin, J. A. Cina, G. R. Fleming, and S. A. Rice, "Fluorescence-detected wave packet interferometry. Time resolved molecular spectroscopy with sequences of femtosecond phase-locked pulses," J. Chem. Phys. 95, 1487-1511 (1991).

10. A. Rebane, C. Sigel, and M. Drobizhev, "Interference between femtosecond pulses observed via time-resolved spontaneous fluorescence," Chem. Phys. Lett. 322, 287-292 (2000).

11. A. K. De and D. Goswami, "Ultrafast pulse-pair control in multiphoton fluorescence laser-scanning microscopy," J. Biomed. Opt. 14, 064018 (2009).

12. A. K. De, D. Roy, and D. Goswami, "Spatio-temporal control in multiphoton fluorescence laser-scanning microscopy," Proc. SPIE 7569, 756929 (2010).

13. S. W. Hell and J. Wichmann, "Breaking the diffraction resolution limit by stimulated emission: stimulated emission depletion microscopy," Opt. Lett. 19, 780-782 (1994).

14. T. A. Klar, S. Jakobs, M. Dyba, A. Egner, and S. W. Hell, "Fluorescence microscopy with diffraction limit broken by stimulated emission," Proc. Natl. Acad. Sci. USA 97, 8206-8210 (2000).

15. M. Dyba, T. A. Klar, S. Jakobs, and S. W. Hell, "Ultrafast dynamics microscopy," Appl. Phys. Lett. 77, 597-599 (2000). 